\title{
Joint Source-Channel Rate Allocation in Parallel Channels
}

\author{
Lingling Pu, Michael W. Marcellin, Ivan Djordjevic, Bane Vasic, and Ali Bilgin \\ Dept. of ECE, The University of Arizona, Tucson, AZ 85721
}

\begin{abstract}
A novel rate-optimal rate allocation algorithm is proposed for parallel transmission of scalable images in multichannel systems. Scalable images are transmitted via fixed-length packets. The proposed algorithm selects a subchannel as well as a channel code rate for each packet, based on the signal-to-noise ratios (SNR) of the subchannels. The resulting scheme provides unequal error protection of source bits. Applications to JPEG2000 transmission show that significant UEP gains are achieved over equal error protection (EEP) schemes.
\end{abstract}

Keywords: Joint source/channel coding, multicarrier/multichannel systems, UEP, JPEG2000

\section{INTRODUCTION}

Parallel channels are often used in multimedia transmission for more reliable communication or more efficient bandwidth usage. They have attracted significant research interest, and yielded many modern communications techniques, such as multicarrier modulation (MCM) or discrete multitone (DMT). ${ }^{1,2}$ Those techniques are well known for their advantages of bandwidth efficiency, excellent ISI performance, and immunity to multi-path fading or time dispersion.

It is well known that joint source-channel coding can improve system performance. Pradhan and Ramchan$\mathrm{dran}^{3}$ propose a multi-resolution modulation to achieve UEP for different resolution layers, but within each layer, equal error protection (EEP) is assigned across subchannels. Turbo-coded OFDM-based interactive video telephony is proposed by Cherriman et. al. ${ }^{4}$ A modulation mode is chosen to maximize throughput while keeping estimated BER lower than a target BER. In addition to the frequency diversity provided by MCM modulation, other diversities, such as space and time diversity, are also studied and utilized in communication systems. An OFDM system with spatial diversity is proposed for image transmission by Song and Liu. ${ }^{5}$ Their scheme uses space-time block codes (STBC) and Reed Solomon codes. Parallel subchannels are assumed to have the same SNR. Source and channel rates are jointly optimized to maximize the expected number of source bits correctly received. Sun et. al. ${ }^{6,7}$ propose the transmission of scalable images over space-time coded OFDM systems. Fast joint source-channel rate allocation is performed for a given average SNR (BER). Chan et. al. ${ }^{8}$ propose to combine multiple description coding and frequency diversity. Parity levels in the FEC-based multiple description coding are optimized for a given SNR.

The above algorithms can be categorized as serial transmission. Such algorithms aim to optimize system performance for a given average SNR (over subchannels). In other words, they try to remove the difference between subchannels to yield the same BER. On the other hand, parallel transmission of a layered source over spectrally shaped channels using MCM is proposed by Zheng and Liu. ${ }^{9}$ Given the constraint on frame power and frame throughput, bit rates and powers are allocated such that channel distortion is minimized. Channel distortion is modelled based on vector quantization for image and video transmission. Only a single bit error within one codeword is assumed in the model. The source layers are predetermined before allocation. Subchannels are ordered by SNR and the earlier (more important) layers select subchannels of higher SNR. The number of subchannels used by each layer is governed by the length of that layer. Resource allocation in multichannel systems are also proposed by Zhao et. al. ${ }^{10,11}$ In their schemes, subchannels are assigned to layered images/videos first, then rates and power levels are allocated jointly between channels.

In this paper, we consider a joint optimal rate allocation for parallel transmission in multiple channels. The proposed system offers UEP for robust image transmission. Source and channel rates are tailored to the SNR differences of subchannels. Scalable sources are transmitted via fixed-length packets. For each packet, the proposed algorithm selects a subchannel among all available subchannels, and chooses a channel code rate, such that the expected length of the correctly received source data is maximized. We verify the proposed algorithm

Visual Communications and Image Processing 2007, edited by Chang Wen Chen, Dan Schonfeld, Jiebo Luo,

Proc. of SPIE-IS\&T Electronic Imaging, SPIE Vol. 6508, 65081A, @ 2007 SPIE-IS\&T · 0277-786X/07/\$18 
in different channel models. Two models are chosen for this purpose. The first one is a model of independent parallel Gaussian channels. This model is chosen because it can represent a non-white Gaussian noise channel or other spectrally shaped channels arising in various applications, such as DMT or MCM modulation, multiple input multiple output (MIMO) systems, and orthogonal frequency division multiplexing (OFDM), etc.

The second model is chosen to demonstrate the suitability of the proposed scheme in a specific channel. To this end, a plastic optical fiber (POF) model is selected. Optical fiber systems have many advantages, such as large bandwidth, low attenuation, secure communications, etc. These advantages make the optical fiber a suitable media for multimedia transmission. POF is a newly developed type of optical fiber. One major advantage of POF over glass fiber is that it is inexpensive and extremely simple to install. POF can be used for Gigabit Ethernet, local area networks (LAN), and high definition television (HDTV) delivery. ${ }^{12-14}$ Randel et. al. ${ }^{15}$ provide results with $1 \mathrm{Gbit} / \mathrm{s}$ transmission in a step-index POF link. In this second example, we apply our algorithm in the POF channel with OFDM modulation.

The paper is organized as follows. The optimization problem is formulated and solved in Section 2. In Section 3, the proposed algorithm is applied to image transmission. The two channel models mentioned above are tested. Section 4 concludes the paper.

\section{OPTIMIZATION PROBLEM}

Suppose that scalable sources are transmitted via fixed-length packets. There are a total of $N$ packets transmitted over $M$ subchannels. Subchannel $m$ transmits $N_{m}$ channel packets, with $\sum_{m=1}^{M} N_{m}=N$. Often $N_{m}=N / M$, but we treat the more general case here. Define the set of available channel code rates as $\mathcal{R}=\left\{r_{1}, r_{2}, \ldots, r_{L}\right\}$ and the set of subchannels as $\mathcal{S}=\left\{s_{1}, s_{2}, \ldots, s_{M}\right\}$. A code rate from $\mathcal{R}$ and a subchannel from $\mathcal{S}$ are chosen for each packet. Ideally, a code rate from $\mathcal{R}$ and a subchannel from $\mathcal{S}$ are chosen for each packet to minimize the end-toend expected distortion. This distortion-based optimization problem is hard to solve in the single channel case, and is even harder for the multi-channel case, since the search space is expanded from $N^{L}$ to $N^{L \times M}$. The number $M$ is usually large, e.g., 128 for a practical OFDM system. In such cases, the expansion of the search space from single channel to multi-channel is prohibitively complex. It has been shown by Hamzaoui et al. ${ }^{16}$ for the single channel case, that the rate-optimal solution provides a good approximation to the distortion-optimal solution for efficient embedded coders. Chande and Farvardin ${ }^{17}$ proposed a rate-optimal solution for fixed-length information block (variable length packets), and Stankovic et al. ${ }^{18}$ extended it to fixed-length packets and proposed a fast algorithm. In this paper, we study the multi-channel optimization problem, and derive a linear time rate-optimal solution.

The problem can be formulated as follows. Let $E_{N}(R)$ denote the expected value of the correctly received source length associated with rate assignment scheme $R . L_{i}(R)$ represents the received source length associated with the event that the first error happens in the $(i+1)^{t h}$ packet under rate assignment scheme $R$, and $P_{i}(R)$ represents the probability of this event. A rate-optimal error protection scheme (EPS) maximizes the expected length of correctly received data,

$$
E_{N}(R)=\sum_{i=1}^{N} P_{i}(R) L_{i}(R)
$$

where

$$
\begin{gathered}
L_{i}(R)=\sum_{j=1}^{i} l\left(r_{k_{j}}\right), \\
P_{i}(R)=p\left(r_{k_{i+1}}, s_{k_{i+1}}\right) \sum_{j=1}^{i}\left(1-p\left(r_{k_{j}}, s_{k_{j}}\right)\right) .
\end{gathered}
$$

and $l\left(r_{k_{j}}\right)$ is the number of source bytes in one packet, which is determined by the channel code rate assigned to the packet. The above expressions assume progressive decoding, and that if one or more errors are detected in one channel packet, this packet and all the following packets are discarded.

We give two lemmas and two corollaries before we derive the optimal solution. 
Lemma 1: Let $R=\left(\left(r_{k_{1}}, s_{k_{1}}\right),\left(r_{k_{2}}, s_{k_{2}}\right), \cdots,\left(r_{k_{N}}, s_{k_{N}}\right)\right) \in(\mathcal{R} \times \mathcal{S})^{N}$ be an $N$-packet EPS. Then

$$
\begin{aligned}
& E_{N}\left[\left(r_{k_{1}}, s_{k_{1}}\right), \cdots,\left(r_{k_{N}}, s_{k_{N}}\right)\right] \\
= & E_{1}\left[\left(r_{k_{1}}, s_{k_{1}}\right)\right]+\left(1-p\left(r_{k_{1}}, s_{k_{1}}\right)\right) \\
& \times E_{N-1}\left[\left(r_{k_{2}}, s_{k_{2}}\right), \cdots,\left(r_{k_{N}}, s_{k_{N}}\right)\right],
\end{aligned}
$$

where $E_{1}\left[\left(r_{k_{1}}, s_{k_{1}}\right)\right]=l\left(r_{k_{1}}\right)\left(1-p\left(r_{k_{1}}, s_{k_{1}}\right)\right)$.

Proof is given in the Appendix.

Lemma 2: If the $(N-1)$-packet EPS $\left(\left(r_{2}^{*}, s_{2}^{*}\right), \cdots,\left(r_{N}^{*}, s_{N}^{*}\right)\right)$ is rate optimal, and if

$$
E_{N}\left[\left(r_{1}^{*}, s_{1}^{*}\right),\left(r_{2}^{*}, s_{2}^{*}\right), \cdots,\left(r_{N}^{*}, s_{N}^{*}\right)\right] \geq E_{N}\left[\left(r_{k}, s_{k}\right),\left(r_{2}^{*}, s_{2}^{*}\right), \cdots,\left(r_{N}^{*}, s_{N}^{*}\right)\right],
$$

for all $r_{k} \in \mathcal{R}$, and $s_{k} \in \mathcal{S}$, then the $N$-packet EPS $\left(\left(r_{1}^{*}, s_{1}^{*}\right),\left(r_{k_{2}}, s_{k_{2}}\right), \cdots,\left(r_{N}^{*}, s_{N}^{*}\right)\right)$ is rate optimal.

Proof is given in the Appendix.

The next two corollaries follow from these lemmas. If the $N$-packet EPS $\left(\left(r_{1}^{*}, s_{1}^{*}\right), \cdots,\left(r_{N}^{*}, s_{N}^{*}\right)\right)$ is rate optimal, then

Corollary 1: For $1 \leq i \leq N-1$, the $(N-i)$-packet EPS $\left(\left(r_{i+1}^{*}, s_{i+1}^{*}\right), \cdots,\left(r_{N}^{*}, s_{N}^{*}\right)\right)$ is rate optimal.

Proof is given in the Appendix.

This result shows that an $N$-packet EPS can be obtained by first calculating a 1-packet EPS (which is trivial), and recursively adding one packet $(N-1)$ times.

Corollary 2: Denote subchannel $s_{n}$ having a smaller bit error rate than $s_{m}$ by $s_{n}>s_{m}$. Then $s_{1}^{*} \geq s_{2}^{*} \geq \cdots \geq s_{N}^{*}$. Proof is given in the Appendix.

From the above two corollaries, the rate-based optimization algorithm can be formulated as follows: Algorithm:

1. Order the $M$ subchannels, s.t. $S C_{1} \geq \cdots \geq S C_{m} \geq \cdots \geq S C_{M}$. Subchannel $S C_{m}$ contains $N_{m}$ packets, and $\sum_{m=1}^{M} N_{m}=N$.

2. Set $i=1, b=M, s_{N-i+1}=S C_{b}$, and $j_{N-i+1}=\arg \max _{k=1, \cdots, L} E_{1}\left[\left(r_{k}, S C_{M}\right)\right]$.

3. $i=i+1$;

If $i=N_{b}+N_{b+1}+\cdots+N_{M}+1$, then $b=b-1, s_{N-i+1}=S C_{b}$;

If $i=N+1$, then $\left\{\left(r_{1}^{*}, s_{1}^{*}\right),\left(r_{2}^{*}, s_{2}^{*}\right), \cdots,\left(r_{N}^{*}, s_{N}^{*}\right)\right\}=\left\{\left(r_{j_{1}}, s_{1}\right),\left(r_{j_{2}}, s_{2}\right), \cdots,\left(r_{j_{N}}, s_{N}\right)\right\}$, and stop.

4. $j_{N-i+1}=\arg \max _{k=1, \cdots, L} E_{i}\left[\left(r_{k}, s_{N-i+1}\right),\left(r_{j_{N-i+2}}, s_{N-i+2}\right), \cdots,\left(r_{j_{N}}, s_{N}\right)\right]$; go to step 3 .

In the above algorithm, the assignment starts from the worst subchannel in step 2 (from Corollary 2). The $N$-packet EPS is derived from a 1-packet EPS, and obtained recursively (from Corollary 1). Whenever each of the packets in the current subchannel has been assigned a channel code rate, the packets in the next worst subchannel will be assigned. The procedure continues until all $N$ packets in all $M$ subchannels are assigned code rates.

\section{APPLICATIONS TO IMAGE TRANSMISSION}

In this section, we apply the proposed algorithm to scalable image transmission. Two channel models are used as examples. The first assumes independent parallel Gaussian channels. The second derives from a plastic optical fiber (POF). The first channel model is chosen as a general example, which can represent various environments such as non-white Gaussian noise channels or spectrally shaped channels. The second channel model is chosen because of its increasing popularity in optical communication systems. Before discussing the two cases individually in subsections 3.2 and 3.3 , we describe the image coding and channel coding techniques used in the experiments. 


\subsection{Source and channel coding}

JPEG2000 coded images are used as source images. JPEG2000 ${ }^{19}$ is the state-of-art image compression standard, which can generate highly scalable codestreams. JEPG2000 divides the subbands from a discrete wavelet transform into rectangular regions called codeblocks. Each bitplane of each codeblock is coded into a number of (compressd) coding passes. Generally, an image is encoded into multiple layers. Each layer contains differing numbers of coding passes from each codeblock. During the encoding procedure, a distortion-rate slope is computed for each coding pass. The distortion-rate slope values characterize the contribution of the coding pass to the quality of the image. Coding passes with larger slope values are more important than those with smaller slope values. A slope threshold is generated to guide the assembly of one layer. All coding passes having slope values larger than the threshold are included in that layer. Hence, these thresholds identify the importance of layers. Consecutive layers have slope thresholds (importance) in descending order. The coding passes in each layer are collected into a number of JPEG2000 packets. These packets are not to be confused with the channel packets described in previous sections.

At the beginning of a codestream, there is a main header, which contains crucial information for the decoder to decode the codestream correctly. Similarly, each JPEG2000 packet has a packet header. If these packet headers are corrupted, the source decoder will not be able to decode the corresponding packets. Thus the optional JPEG2000 packed packet header marker segments (PPM) are used in our experiments to move all JEPG2000 packet headers to the main header. The main header (including the relocated packet headers) is then assigned the lowest code rate (highest protection).

In what follows, we consider the case where multiple images are transmitted through a common channel. These images may be entirely unrelated. However, in Part III of the JPEG2000 standard (referred to as Motion JPEG2000), each frame of a video sequence is compressed independently. Thus, the multiple images transmitted through the channel (using our proposed system) could be frames of a video.

We examine two methods to transmit multiple images. We first consider transmitting each image with an equal number of channel packets. For example, if 256 packets are used to transmit 4 images, each image occupies 64 packets. In this case the total available resources are divided equally. We next consider multiplexing the images together and adjusting the available resources between the images according to their characteristics. The multiplexing is done by combining all the layers from all the images. All the main headers are also combined and placed at the beginning of the resulting codestream. Since each layer is associated with a threshold slope value, all the layers (from all images) can be ordered by their threshold slope values. The combined codestream can be viewed as a large "pseudo" image.

Packetization and rate allocation can then be performed on the "pseudo" image using the algorithm described in Section 2 above. At the channel output, the "pseudo" image is split into individual images, each of which is decoded separately by a JPEG2000 decoder. If one or more errors are detected in one channel packet, this packet and all the following packets are discarded. This procedure is based on the assumption that later layers cannot be decoded without the reception of earlier layers. This assumption is not strictly satisfied since an error in an image will not affect the decoding of another image. Nevertheless, we make the assumption here for the sake of simplicity.

Rate-compatible punctured turbo codes $(\mathrm{RCPT})^{20}$ are employed as channel codes. A rate $1 / 3$ parallel concatenated convolutional code with generator polynomial $(33,31)$ is chosen as the encoder. A maximum number of 20 iterations are used for decoding. Channel packets of length 512 bytes are used. Four bytes are reserved for 32-bit CRC, which is used for error detection following turbo decoding. One byte is reserved for transmitting side information such as the turbo code rate. Code rates are chosen from the set $\mathcal{R}=\{8 / 9,8 / 10, \cdots, 8 / 23,8 / 24\}$. The cardinality of this set is 16 . The following subsections will discuss transmission for two different channel models.

\subsection{Independent Parallel Gaussian Channels}

As a first example, independent parallel Gaussian channels are assumed. BPSK modulation is used. Four greylevel $512 \times 512$ images are used as source images: Lena, Goldhill, Whitehouse, and Lighthouse. We choose 32 AWGN channels with equally spaced SNRs between $1.0 \mathrm{~dB}$ and $3.0 \mathrm{~dB}$. 
Table 1. Average MSE and PSNR for $1.0 \mathrm{bpp} /$ source and parallel Gaussian channels.

\begin{tabular}{|c|c|c|}
\hline & EEP & UEP \\
\hline Lena & 13.72 & 48.64 \\
\hline Goldhill & 34.30 & 74.89 \\
\hline Whitehouse & 238.44 & 186.37 \\
\hline Lighthouse & 489.96 & 177.61 \\
\hline Average MSE & 194.11 & 121.88 \\
\hline Average PSNR & 25.25 & 27.27 \\
\hline
\end{tabular}

Multiple source images are multiplexed together using the method described in 3.1. Then the rate allocation algorithm described in Section 2 is used to assign a channel and code rate to each packet. For comparison purposes, an EEP scheme is tested. In the EEP scheme, the images are not multiplexed. Rather, each source image is assigned the same number of channel packets. For example, suppose that a total of 256 packets are to be transmitted through 32 subchannels, with each subchannel transmitting 8 packets. Assume there are four source images. Each image is then allocated two packets from each subchannel. All the packets are protected by the same code, with a code rate equal to the average code rate used in the UEP scheme described before. In both the schemes, headers are protected by a code of rate $1 / 3$.

Simulation results are listed in Table 1. A total number of $N=256$ packets are used for an average rate of $1.0 \mathrm{bpp} /$ source, including all header and parity info. The resulting MSE values (from 1000 simulations) are reported for each image. The average MSE over all images is reported at the bottom of the table. The average PSNR is obtained by converting the average MSE. By means of the rate allocation method proposed in this paper, resources are distributed to minimize the average MSE over all source images at the output of the noisy channels. By comparing the results from UEP and EEP, it can be concluded that proposed rate allocation significantly improves the average system performance. Intuitively, resources are taken from the "easy" images, to improve the quality of the "difficult" images in a way so that average quality is maximized. An added benefit is that the quality variance is reduced significantly from image to image.

\subsection{Plastic Optical Fiber Channel}

In addition to the general model of independent parallel Gaussian channels discussed in the previous section, a graded-index plastic optical fiber (GI-POF) channel model with OFDM modulation is considered as the second example.

The impulse response of GI-POF can be modelled ss $^{21}$

$$
h(t)=\frac{1}{\sqrt{2 \pi \alpha^{2}}} \exp \left(-\frac{t^{2}}{2 \alpha^{2}}\right) .
$$

Its transfer function (Fourier transform) is

$$
H(\omega)=\exp \left(-\frac{\alpha^{2} \omega^{2}}{2}\right)
$$

where $\alpha=\sqrt{2 \ln 2} T_{b} / 2 \pi f_{n}, T_{b}$ is the pulse coded modulation (PCM) bit time, and $f_{n}$ is the channel bandwidth normalized to the PCM data rate.

OFDM modulation ${ }^{22}$ is considered. For completeness, a brief introduction on OFDM is presented below. The information sequence is subdivided into $K$ groups (subchannels). For coded OFDM systems, information bits are first passed through a channel encoder for each subchannel. The OFDM modulator consists of $K$ independent subchannels. $K$ signal points, $X_{k}$, are chosen from certain constellations according to any phase shift keying (PSK) or quadrature amplitude modulation (QAM) signaling set (symbol mapping). BPSK modulation is tested in our experiments. Those symbols can be viewed as values of the discrete Fourier transform (DFT) of an OFDM signal $x(t)$. A cyclic prefix is appended to avoid intersymbol interference (ISI). The signal is transmitted through some channel after $\mathrm{D} / \mathrm{A}$ conversion. The received signal may be expressed as $r(t)=x(t) \star h(t)+n(t)$, where $\star$ represents convolution, and $n(t)$ is additive noise corrupting the signal. At the receiver end, inverse procedures 
Table 2. Average MSE and PSNR for $1.0 \mathrm{bpp} /$ source and GI-POF channel.

\begin{tabular}{|c|c|c|}
\hline & EEP & UEP \\
\hline Lena & 12.47 & 41.16 \\
\hline Goldhill & 31.11 & 67.81 \\
\hline Whitehouse & 225.90 & 158.07 \\
\hline Lighthouse & 469.29 & 172.15 \\
\hline Average MSE & 184.69 & 109.80 \\
\hline Average PSNR & 25.47 & 27.72 \\
\hline
\end{tabular}

are taken to recover the original information symbols, $X_{k}$. For a coded system, the output bits are decoded by a channel decoder.

In our experiments, we employ the GI-POF channel. The non-flat shape of the transfer function of the POF channel causes the subchannels to have different channel gains. In particular, the signal $x(t)$ is convolved with the impulse response of the channel (see Equation 5). Equivalently, the subchannel symbols $X_{k}$ are multiplied by the transfer function of the channel (see Equation 6). We assume the additive noise in the system is AWGN, and SNR is denoted by $\gamma_{0}$. From Equation 6 , the gain $\rho_{k}$ of each subchannel can be estimated. More importantly, the equivalent SNR of each subchannel can be estimated as $\gamma_{k}=\rho_{k}^{2} \gamma_{0}$. The same image sources are used as in the previous example. In the experiments, the OFDM system has 128 subchannels. A channel SNR of $\gamma_{0}=3 \mathrm{~dB}$ and $f_{n}=1.3$ are selected. The bit rate of the system is $2.5 \mathrm{~Gb} / \mathrm{s}$, and a total of 256 packets are transmitted. The results are listed in Table 3.3. It can be seen from the table that UEP provides about $2.2 \mathrm{~dB}$ gain.

\section{CONCLUSION}

In this paper, a multi-channel rate allocation algorithm is proposed. A fast algorithm assigns unequal error protection to each segment of a source to maximize the expected value of the correctly received source length. Differences between subchannels are utilized. The algorithm can be applied to many systems that suffer from nonideal channels. Applications to JPEG2000 transmission shows the superiority of the proposed algorithm by exploiting the differences between subchannels instead of using the average subchannel SNR. Significant UEP gains are achieved over EEP schemes.

\section{APPENDIX}

\subsection{Proof of Lemma 1}

$$
\begin{aligned}
& E_{N}\left[\left(r_{k_{1}}, s_{k_{1}}\right), \cdots,\left(r_{k_{N}}, s_{k_{N}}\right)\right] \\
= & l\left(r_{k_{1}}\right) \sum_{i=1}^{N} P_{i}(R)+\sum_{i=2}^{N} P_{i}(R) \sum_{j=2}^{i} l\left(r_{k_{j}}\right) \\
= & l\left(r_{k_{1}}\right)\left(1-p\left(r_{k_{1}}, s_{k_{1}}\right)\right)+\sum_{i=2}^{N} P_{i}(R) \sum_{j=2}^{i} l\left(r_{k_{j}}\right) \\
= & l\left(r_{k_{1}}\right)\left(1-p\left(r_{k_{1}}, s_{k_{1}}\right)\right)+\left(1-p\left(r_{k_{1}}, s_{k_{1}}\right)\right) \\
& \times E_{N-1}\left[\left(r_{k_{2}}, s_{k_{2}}\right), \cdots,\left(r_{k_{N}}, s_{k_{N}}\right)\right] .
\end{aligned}
$$

\subsection{Proof of Lemma 2}

$$
\begin{aligned}
& E_{N}\left[\left(r_{1}^{*}, s_{1}^{*}\right),\left(r_{2}^{*}, s_{2}^{*}\right), \cdots,\left(r_{N}^{*}, s_{N}^{*}\right)\right] \\
\geq & \left(1-p\left(r_{k_{1}}, s_{k_{1}}\right)\right)\left[l\left(r_{k_{1}}\right)+E_{N-1}\left[\left(r_{2}^{*}, s_{2}^{*}\right), \cdots,\left(r_{N}^{*}, s_{N}^{*}\right)\right]\right. \\
\geq & \left(1-p\left(r_{k_{1}}, s_{k_{1}}\right)\right)\left[l\left(r_{k_{1}}\right)+E_{N-1}\left[\left(r_{k_{2}}, s_{k_{2}}\right), \cdots,\left(r_{k_{N}}, s_{k_{N}}\right)\right]\right. \\
= & E_{N}\left[\left(r_{k_{1}}, s_{k_{1}}\right),\left(r_{k_{2}}, s_{k_{2}}\right), \cdots,\left(r_{k_{N}}, s_{k_{N}}\right)\right] .
\end{aligned}
$$




\subsection{Proof of Corollary 1}

$$
\begin{gathered}
E_{N}\left[\left(r_{1}^{*}, s_{1}^{*}\right),\left(r_{2}^{*}, s_{2}^{*}\right), \cdots,\left(r_{N}^{*}, s_{N}^{*}\right)\right] \\
\geq E_{N}\left[\left(r_{1}^{*}, s_{1}^{*}\right),\left(r_{k_{2}}, s_{k_{2}}\right), \cdots,\left(r_{k_{N}}, s_{k_{N}}\right)\right],
\end{gathered}
$$

which implies

$$
\begin{aligned}
& E_{1}\left[\left(r_{1}^{*}, s_{1}^{*}\right)\right]+\left(1-p\left(r_{1}^{*}, s_{1}^{*}\right)\right) E_{N-1}\left[\left(r_{2}^{*}, s_{2}^{*}\right), \cdots,\left(r_{N}^{*}, s_{N}^{*}\right)\right] \\
\geq & E_{1}\left[\left(r_{1}^{*}, s_{1}^{*}\right)\right]+\left(1-p\left(r_{1}^{*}, s_{1}^{*}\right)\right) \\
\times & E_{N-1}\left[\left(r_{k_{2}}, s_{k_{2}}\right), \cdots,\left(r_{k_{N}}, s_{k_{N}}\right)\right] .
\end{aligned}
$$

This in turn yields

$$
\begin{gathered}
E_{N-1}\left[\left(r_{2}^{*}, s_{2}^{*}\right), \cdots,\left(r_{N}^{*}, s_{N}^{*}\right)\right] \\
\geq E_{N-1}\left[\left(r_{k_{2}}, s_{k_{2}}\right), \cdots,\left(r_{k_{N}}, s_{k_{N}}\right)\right] .
\end{gathered}
$$

Similarly, we can get

$$
\begin{gathered}
E_{N-i}\left[\left(r_{i+1}^{*}, s_{i+1}^{*}\right), \cdots,\left(r_{N}^{*}, s_{N}^{*}\right)\right] \\
\geq E_{N-i}\left[\left(r_{k_{i+1}}, s_{k_{i+1}}\right), \cdots,\left(r_{k_{N}}, s_{k_{N}}\right)\right] .
\end{gathered}
$$

\subsection{Proof of Corollary 2}

From Corollary 1, we have

$$
E_{2}\left[\left(r_{N-1}^{*}, s_{N-1}^{*}\right),\left(r_{N}^{*}, s_{N}^{*}\right)\right] \geq E_{2}\left[\left(r_{N-1}^{*}, s_{N}^{*}\right),\left(r_{N}^{*}, s_{N}^{*}\right)\right] .
$$

By Equation 4, we obtain

$$
\begin{gathered}
E_{1}\left[\left(r_{N-1}^{*}, s_{N-1}^{*}\right)\right]+\left(1-p\left(r_{N-1}^{*}, s_{N-1}^{*}\right)\right) E_{1}\left[\left(r_{N}^{*}, s_{N}^{*}\right)\right] \\
\geq E_{1}\left[\left(r_{N-1}^{*}, s_{N}^{*}\right)\right]+\left(1-p\left(r_{N-1}^{*}, s_{N}^{*}\right)\right) E_{1}\left[\left(r_{N}^{*}, s_{N}^{*}\right)\right],
\end{gathered}
$$

which indicates that

$$
\begin{aligned}
& E_{1}\left[\left(r_{N-1}^{*}, s_{N}^{*}\right)\right]-E_{1}\left[\left(r_{N-1}^{*}, s_{N-1}^{*}\right)\right] \\
\leq & \left(p\left(r_{N-1}^{*}, s_{N}^{*}\right)-p\left(r_{N-1}^{*}, s_{N-1}^{*}\right)\right) E_{1}\left[\left(r_{N}^{*}, s_{N}^{*}\right)\right] .
\end{aligned}
$$

If $s_{N}^{*}>s_{N-1}^{*}$, then Equation 10 has $R H S<0$ and $L H S>0$, which is a contradiction. Thus, we must have $s_{N-1}^{*} \geq s_{N}^{*}$. Similarly,

$$
\begin{gathered}
E_{3}\left[\left(r_{N-2}^{*}, s_{N-2}^{*}\right),\left(r_{N-1}^{*}, s_{N-1}^{*}\right),\left(r_{N}^{*}, s_{N}^{*}\right)\right] \\
\geq E_{3}\left[\left(r_{N-2}^{*}, s_{N-1}^{*}\right),\left(r_{N-1}^{*}, s_{N-1}^{*}\right),\left(r_{N}^{*}, s_{N}^{*}\right)\right],
\end{gathered}
$$

which yields

$$
\begin{aligned}
& E_{1}\left[\left(r_{N-2}^{*}, s_{N-2}^{*}\right)\right]+\left(1-p\left(r_{N-2}^{*}, s_{N-2}^{*}\right)\right) \\
\times & E_{2}\left[\left(r_{N-1}^{*}, s_{N-1}^{*}\right),\left(r_{N}^{*}, s_{N}^{*}\right)\right] \geq E_{1}\left[\left(r_{N-2}^{*}, s_{N-1}^{*}\right)\right] \\
+ & \left(1-p\left(r_{N-2}^{*}, s_{N-1}^{*}\right)\right) E_{2}\left[\left(r_{N-1}^{*}, s_{N-1}^{*}\right),\left(r_{N}^{*}, s_{N}^{*}\right)\right] .
\end{aligned}
$$

This leads to

$$
\begin{aligned}
& E_{1}\left[\left(r_{N-2}^{*}, s_{N-1}^{*}\right)\right]-E_{1}\left[\left(r_{N-2}^{*}, s_{N-2}^{*}\right)\right] \\
\leq & \left(p\left(r_{N-2}^{*}, s_{N-1}^{*}\right)-p\left(r_{N-2}^{*}, s_{N-2}^{*}\right)\right) \\
\times & E_{2}\left[\left(r_{N-1}^{*}, s_{N-1}^{*}\right),\left(r_{N}^{*}, s_{N}^{*}\right)\right] .
\end{aligned}
$$

By the same argument as above, we obtain $s_{N-2}^{*} \geq s_{N-1}^{*}$. The same method can be used to show that $s_{i-1}^{*} \geq s_{i}^{*}$, for $2 \leq i \leq N$. 


\section{REFERENCES}

1. J. Bingham, "Multicarrier modulation for data transmission: An idea whose time has come," IEEE Communications Magazine 28, pp. 5-14, May 1990.

2. I. Kalet, "The multitone channel," IEEE Trans. on Communications 37, pp. 119-124, Feb. 1989.

3. S. Pradhan and K. Ramchandran, "Optimized embedded multicarrier modulation for efficient delivery of layered video data," in Proceedings, IEEE International Conference on Communications, 2, pp. 1009-1012, June 1998.

4. P. Cherriman, T. Keller, and L. Hanzo, "Subband-adaptive turbo-coded OFDM-based interactive video telephony," IEEE Trans. on Circuits, Systems, Video Technology 12, pp. 829-839, Oct. 2002.

5. J. Song and K. Liu, "Robust progressive image transmission over OFDM systems using space-time block code," IEEE Trans. on Multimedia 4, pp. 394-406, Sep. 2002.

6. Y. Sun, Z. Xiong, and X. Wang, "Scalable image transmission over differentially space-time coded OFDM systems," in Proceedings, Global Telecommunications Conference, pp. 379-383, Nov. 2002.

7. Y. Sun and Z. Xiong, "Progressive image transmission over space-time coded OFDM-based MIMO systems with adaptive modulation," IEEE Trans. on Mobile Computing, pp. 1016-1028, Aug. 2006.

8. Y. Chan, P. Cosman, and L. Milstein, "A cross-layer diversity technique for multicarrier OFDM multimedia networks," IEEE Trans. on Image Processing 15, pp. 833-847, Apr. 2006.

9. H. Zheng and K. Liu, "Robust image and video transmission over spectrally shaped channels using multicarrier modulation," IEEE Trans. on Multimedia 1, pp. 88-103, Mar. 1999.

10. S. Zhao, Z. Xiong, and X. Wang, "Optimal resource allocaiton for wireless video over CDMA networks," IEEE Trans. on Mobile Computing 4, pp. 56-67, Jan./Feb. 2006.

11. S. Zhao, Z. Xiong, W. X., and J. Hua, "Progressive video delivery over wideband wireless channels using space-time differentially coded OFDM systems," IEEE Trans. on Mobile Computing 5, pp. 303-316, Apr. 2006.

12. R. S. P. Mederer, F.and Jäger, H. Unold, M. Kicherer, K. Ebeling, M. Naritomi, and R. Yoshida, "Multi-Gb/s graded-index POF data link with butt-coupled single-mode InGaAs VCSEL," IEEE photonics technology letters 12, pp. 199-201, Feb. 2000.

13. T. Matsuoka, T. Ito, and T. Kaino, "First plastic optical fibre transmission experiment using 520nm LEDs with intensity modulation/direct detecion," IEE Electronics Letters 36, pp. 1836-1837, Oct. 2000.

14. Available at http://www.technologynewsdaily.com/node/2927.

15. S. Randel, S. Lee, B. Spinnler, F. Breyer, H. Rohde, J. Walewski, A. Koonen, and A. Kirstädter, "1 Gbit/s transmission with $6.3 \mathrm{bit} / \mathrm{s} / \mathrm{hz}$ spectral efficency in a $100 \mathrm{~m}$ standard $1 \mathrm{~mm}$ step-index plastic optical fibre link using adaptive multiple sub-carrier modulation," in 32nd European Conference on Optical Communication, pp. 41-42, Sep. 2006.

16. R. Hamzaoui, V. Stankovic, and Z. Xiong, "Rate-based versus distortion-based optimal joint source-channel coding," in Proceedings, Data Compression Conference, pp. 63-72, (Snowbird, Utah), April 2002.

17. V. Chande and N. Farvardin, "Progressive transmission of images over memoryless noisy channels," IEEE Journal on Selected Areas in Communications 18, pp. 850-860, Jun. 2000.

18. V. Stankovic, R. Hamzaoui, and D. Saupe, "Fast algorithm for rate-based optimal error protection of embedded codes," IEEE Trans. on Communications 51, pp. 1788-1795, Nov. 2003.

19. D. Taubman and M. Marcellin, JPEG2000: Image compression fundamentals, standards and practice, Kluwer Academic Publishers, 2002.

20. S. Benedetto, D. Divsalar, G. Montorsi, and F. Pollara, "A soft-input soft-output maximum a poteriori (MAP) module to decode parallel and serial concatenated codes," TDA Progress Report, pp. 42-127, Nov. 1996.

21. B.-G. Shin, J.-H. Park, and J.-J. Kim, "Low-loss, high-bandwidth graded-index plastic optical fiber fabricated by the centrifugal deposition method," Applied Physics Letters 82 (26), pp. 4645-4647, Jun. 2003.

22. R. Prasad, OFDM for wireless communications systems, Artech House, Inc., 2004. 